\title{
PROBLEM OF PROFILE MATCHING IN SPHERICITY MEASUREMENTS BY THE RADIAL METHOD
}

\section{Dariusz Janecki, Stanisław Adamczak, Krzysztof Stępień}

Kielce University of Technology, Institute of Mechanical Technology and Metrologu, Al. 1000-lecia P. P. 7, 25-314 Kielce, Poland (djanecki@tu.kielce.pl, adamczak@tu.kielce.pl, $₫$ kstepien@tu.kielce.pl, +48 41342 4477)

\begin{abstract}
At the Kielce University of Technology a new concept of accurate measurements of sphericity deviations of machine parts has been developed. The concept is based upon measurement of roundness profiles in many clearly defined cross-sections of the workpiece. Measurements are performed with the use of typical radial measuring instrument equipped with a unit allowing accurate positioning of the ball. The developed concept required finding a solution to numerous problems relating to the principle of the radial measurement. One of the problems to be solved was matching of measured roundness profiles. The paper presents an outline of the developed concept of sphericity measurement, a mathematical model of profile matching and results of the verification of the model.
\end{abstract}

Keywords: sphericity, measurement, profiles, matching, mathematical model.

(C) 2012 Polish Academy of Sciences. All rights reserved

\section{Introduction}

The evaluation of geometrical surface structure is a crucial matter in modern manufacturing processes [1]. Among elements utilized in mechanical engineering a particularly important group constitutes spherical machine parts that are used mainly in the bearing industry. Measurement of such elements are usually performed with the use of radial instruments [2]. In such instruments a ball is placed on the measuring table, which, depending on the type, can rotate or it is immovable. If the table is immovable, then a measuring sensor can rotate [3-4]. Information on other methods of sphericity measurement are quite poor. Gleason and Schwenke propose in [5] to apply a classical 3-point method to measure spherical elements. Another group of methods that are used to measure deviations of spherical elements are optical methods. Udupa et al in the work [6] apply a confocal scanning optical microscope. Bartl et al in the work [7] apply a special-purpose sphere interferometer in a combination with a stitching approach. Halkaci et al in the work [8] use an image-processing method to evaluate form errors of semi-spherical EDM tools. Chen in the work [9] applies an optical microscope to measure spherical balls of miniaturized coordinate measuring probes.

Another method that is tested in the area of measurements of spherical elements is coordinate metrology [10-11]. However, existing coordinate measuring machines do not offer a measurement accuracy that would be satisfying for manufacturers of precise machine parts (for example the bearing industry).

In past practice form errors of spherical machine parts have been evaluated on the basis of results of measurements of roundness profiles in a few selected cross-sections of the specimen. Such approach allows only a rough evaluation of form errors, particularly if there are local irregularities on the surface. This is the reason why at the Kielce University of 
Technology a concept has been developed which allows to measure roundness profiles along trajectories selected in a way assuring accurate coverage of the surface with a net of sampling points. It can be achieved, for example, by performing measurements along equally distributed cross-sections in two orientations of the sphere rotated in relation to each other at the straight angle around a vertical axis (coinciding a spindle axis of an instrument). Such measurement is possible to perform with the use a typical radial instrument equipped with a unit that enables positioning of the inspected sphere [12]. The proposed concept required to solve numerous problems related to the assumed measurement principle, including matching of measured roundness profiles.

\section{The principle of the sphericity evaluation by the radial method}

The concept of the research work on accurate measurements of sphericity deviations has been developed at the Kielce University of Technology on the basis of analysis of scientific literature and with the use of researchers' experience in the field of accurate measurements of roundness and cylindricality deviations. The first stage of the research work was solving of numerous theoretical problems relating to the developed concept.

The theoretical part of the research work included:

- a definition of an example of a spherical surface;

- the choice of a relevant measuring strategy;

- generation of profiles and their matching;

- filtration of profiles;

- determination of the reference sphere;

- determination of sphericity parameters;

- an approximation of the measured profile with a surface.

Operations necessary to evaluate form errors of spherical elements with the use of measurement data obtained from a radial measuring instrument are shown in Fig. 1.

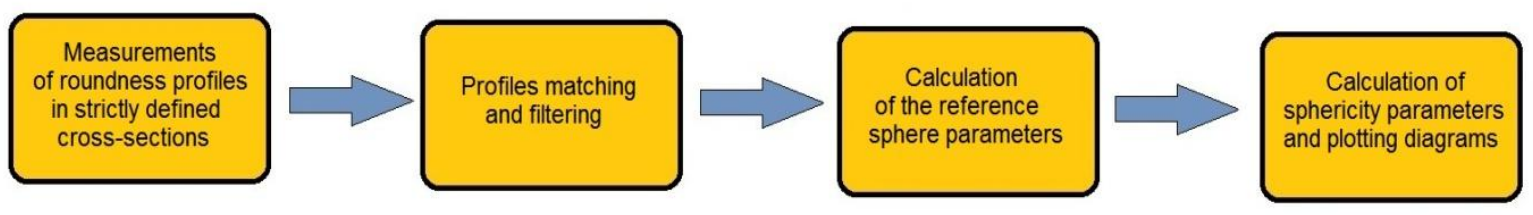

Fig. 1. Stages of data processing in the evaluation of sphericity by the radial method.

One of the most important problems to be solved was correct matching of measured roundness profiles. The solution of the problem of profile matching is necessary if one takes into account the assumed measurement method, i.e. the radial method. Radial instruments do not measure the real value of a radius of a specimen, but variations of the radius in relation to a certain initial value, which is generally different in different sections. In the assumed measurement concept, after a measurement of the roundness profile in a given section, the sensor is being moved away from the element surface, and next it touches the surface during the measurement of another cross-section. Depending on the measurement strategy, profiles cross one another in a different number of measuring points on the investigated surface. Due to the relative character of the radial method, the radii values in points of profile crossings, indicated by the sensor when different sections are measured, are not equal to one another. Thus, it is necessary to match them. It can be done because in the profile crossings the radii values should be equal to each other.

There were many different measuring strategies analyzed during the research work. Examples of analyzed strategies are shown in Fig. 2. 

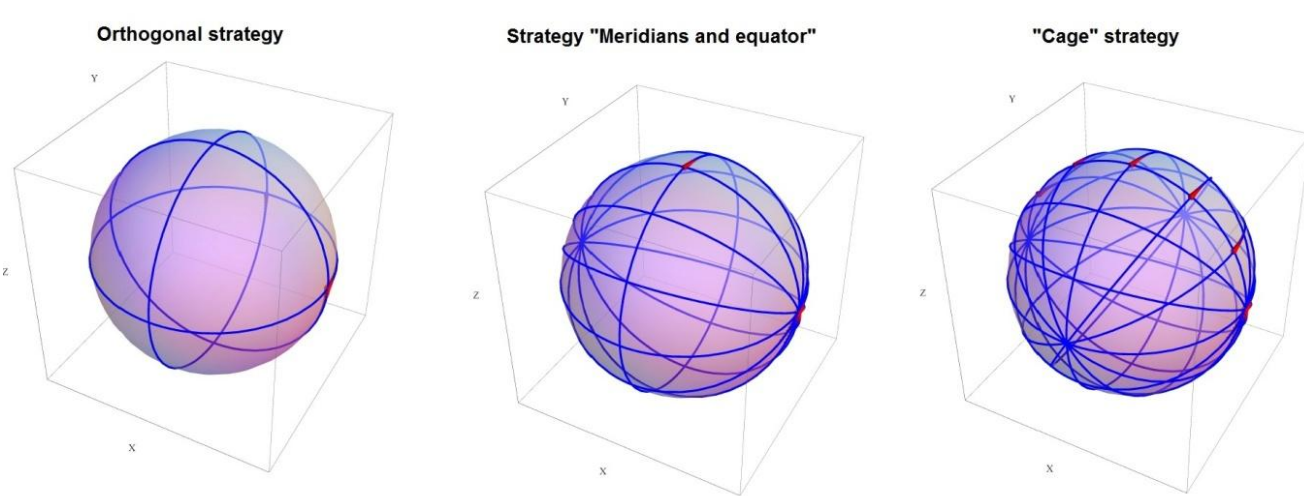

Fig. 2. Examples of analyzed measuring strategies.

Taking into account results of performed analyses and capabilities of the applied measuring instrument it was assumed that the preferred measuring strategy will be the socalled "Cage" strategy. In this strategy measurements are performed along equally distributed cross-sections in two orientations of the sphere rotated mutually at a the straight angle around a vertical axis (coinciding a spindle axis of an instrument).

\section{Mathematical model of profile matching}

\subsection{Calculation of coordinates of profile crossings}

As it was shown in Fig. 2, numerous strategies can be applied to measure sphericity deviations. The strategies differ in the number of measured roundness profiles that cross each other. It is obvious that the number of crossing points and their location depend on the strategy that is applied. Before conducting profile matching it is necessary to calculate coordinates of the crossing points. This problem has been solved with the use of Mathematica software.

Let us assume that a 3D Cartesian coordinate system XYZ is associated with the investigated element (see Fig. 3).

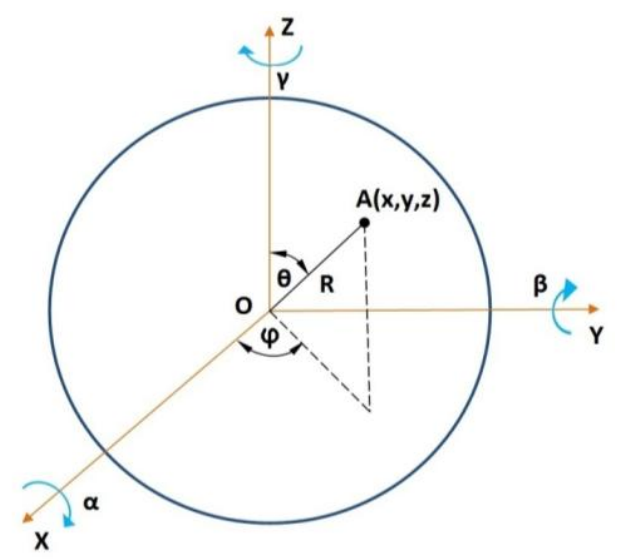

Fig. 3. Coordinate system used in the solution of the problem of profile matching.

It is obvious that relevant axes of the system XYZ will change their orientations if the sphere rotates. In order to solve the problem of determination of coordinates of scanning trajectory crossings one more Cartesian coordinate system will be useful, that is associated with the measuring instrument. This system will be denoted by X'Y'Z'. In a mathematical description of orientation of individual points on the sphere, the application of spherical 
coordinates $R, \theta, \varphi$ is convenient, where $\theta \in<0, \pi>$ and $\varphi \in<0,2 \pi>$. The coordinate $R$ is obviously the distance of a given point from the origin of the system, $\theta$ is the angle between the Z-axis and a line connecting the point and the origin of the system, and $\varphi$ is the angle between the projection of the point onto the XY plane and the X-axis [14]. The change of the sphere orientation from its initial state can be described by three angular values $(\alpha, \beta, \gamma)$ that denote a rotation of the element around the $\mathrm{X}, \mathrm{Y}$ and $\mathrm{Z}$-axis of the assumed coordinate system, respectively.

Let us consider two orientations of the sphere given by two sets of angles $\left(\alpha_{1}, \beta_{1}, \gamma_{1}\right)$ and $\left(\alpha_{2}, \beta_{2}, \gamma_{2}\right)$. In order to solve the problem of profile matching, the coordinates of trajectory crossings and angular coordinates of the crossings in the system of the instrument should be determined. It can be carried out by defining a unit vector parallel to the Z'-axis in the system of the sphere applying a relevant rotation matrix. The coordinates of this vector can be calculated with the use of the following instructions of the Mathematica software:

$\mathrm{A}^{\mathrm{T}} .\{0,0,1\}$

and the rotation matrix $\mathrm{A}$ is given by the instruction:

$A=\operatorname{Rz}[\beta] \cdot \operatorname{Ry}[\alpha] \cdot \operatorname{Rz}[-\beta] \cdot \operatorname{Rz}[\gamma]$;

The vector, whose coordinates were calculated, is perpendicular to the plane of the measured profile. Thus, vectors $v_{1}$ and $v_{2}$ perpendicular to planes of both profiles can be calculated in the following way:

$\mathrm{v} 1=\mathrm{A}^{\mathrm{T}} .\{0,0,1\} / .\{\alpha \rightarrow \alpha 1, \beta \rightarrow \beta 1, \gamma \rightarrow \gamma 1\}$;

$\mathrm{v} 2=\mathrm{A}^{\mathrm{T}} \cdot\{0,0,1\} / .\{\alpha \rightarrow \alpha 2, \beta \rightarrow \beta 2, \gamma \rightarrow \gamma 2\}$;

The vector indicating the crossing point of the profiles is perpendicular to both vectors $v_{1}$ and $v_{2}$. Therefore, it can be determined by a vector product of these vectors $v_{o}$, which is executed by the following Mathematica instruction:

$\mathrm{vo}=\mathrm{v} 1 \mathrm{x}_{\mathrm{v} 2}$

The angular location $\varphi_{1}^{\prime}$ and $\varphi_{2}^{\prime}$ of the crossing points in systems associated with the instrument during measurements can be calculated as follows:

tmp $=$ Cart 2 Spheric $[(A / .\{\alpha \rightarrow \alpha 1, \beta \rightarrow \beta 1, \gamma \rightarrow \gamma 1\})$. vo ];

The first element is the vector norm, the second is the value of the angle $\theta$ at which the measurement was conducted and the third is the sought angle $\varphi^{\prime}$.

On the basis of the described methodology a function GetCros SectionPhase has been developed in Mathematica software that calculates coordinates $\varphi^{\prime}$ of crossing points of two profiles and the length of the vector $v_{o}$. It is obvious that if this length is equal to 1 , then the vectors $v_{1}$ and $v_{2}$ are orthogonal and if this length is small then the sections are close to each other. The code of the function GetCrosSectionPhase is given below:

GetCrosSectionPhase [m1_List, m2_List]: =Module $[\{\mathrm{A} 1, \mathrm{~A} 2, \mathrm{v} 1, \mathrm{v} 2, \mathrm{vo}, \varphi 1, \varphi 2\}$,

$\mathrm{A} 1=\mathrm{A} /$. sub $[\mathrm{m} 1]$;

$\mathrm{A} 2=\mathrm{A} /$. sub $[\mathrm{m} 2]$

$\mathrm{V} 1=\mathrm{A} 1^{\mathrm{T}} \cdot\{0,0,1\}$

$\mathrm{v} 2=\mathrm{A} 2^{\mathrm{T}} \cdot\{0,0,1\}$;

$\mathrm{VO}=\mathrm{V} 1 \mathrm{x}_{\mathrm{V}} 2 / / \mathrm{Full}$ Simplify;

$\mathrm{v} 1=\mathrm{A} 1 \cdot \mathrm{vo}$

$\mathrm{v} 2=\mathrm{A} 2 \cdot \mathrm{vo}$

$\varphi 1=$ Cart 2 Spheric [v1] [ [3] ];

$\varphi 2=$ Cart2Spheric [v2] [ [3] ];

$\{\varphi 1, \varphi 2$, Norm [vo] $\}]$

Note that if GetCrosSectionPhase $[\mathrm{m} 1, \mathrm{~m} 2]$ provides coordinates of a certain crossing point, then GetCrosSectionPhase [m2, m1] gives coordinates of the opposite point (the vector $v_{o}$ will have an opposite sense, then). 


\subsection{Profile matching}

Let $\left(\alpha_{1}, \beta_{1}, \gamma_{1}\right), i=1, \ldots, N$ be the set of orientations of the measured sphere. There is a scanning trajectory $\left(\varphi_{i}\left(\varphi^{\prime}\right), \theta_{i}\left(\varphi^{\prime}\right)\right), \varphi^{\prime} \in(0,2 \pi)$ defined for each orientation. Each measured profile has its random mean value $c_{i}$ and eccentricity $a_{i} \cos \varphi^{\prime}+b_{i} \sin \varphi^{\prime}$, so the measured profile is given by:

$$
\begin{aligned}
& r_{\text {meas }, i}\left(\varphi^{\prime}\right)=R_{\text {sphere }}\left(\theta_{i}\left(\varphi^{\prime}\right), \varphi\left(\varphi^{\prime}\right)\right)-a_{i} \cos \varphi^{\prime}-b_{i} \sin \varphi^{\prime}-c_{1}-\varepsilon_{i}= \\
& =r_{\text {sphere }, i}\left(\varphi^{\prime}\right)-\Theta\left(\varphi^{\prime}\right)^{T} p_{\text {match }, i}-\varepsilon_{i},
\end{aligned}
$$

where:

- $\varepsilon_{i}\left(\varphi^{\prime}\right)$ are errors of the instrument, errors of measurements of the angles of the sphere orientations, etc.;

- $r_{\text {sphere } i}\left(\varphi^{\prime}\right)$ is the real profile in a given $i$ cross section;

- $\quad p_{\text {match }, i}=\left[\begin{array}{lll}a_{i} & b_{i} & c_{i}\end{array}\right]^{T}$ are the parameters of the matching of Section 1;

- $\Theta\left(\varphi^{\prime}\right)=\left[\begin{array}{lll}\cos \varphi^{\prime} & \sin \varphi^{\prime} & 1\end{array}\right]^{T}$ Profile matching is actually such choice of values of parameters $a_{i}$; $b_{i}, c_{i}$ that assures possibly the least difference between radii in points of crossings of the scanning trajectory. It is obvious that for the first profile these parameters can be set to zero.

It is easy to notice that profile matching cannot be performed for a method consisting of measurements of two profiles, because there are only two crossing points and two equations, whereas there are three unknown parameters then.

A similar case is when there are many profiles to be measured, but all of them go through two points that are located symmetrically in relation to the origin of the sphere (poles). Then, $N-1$ equations can be formulated for each pole, which gives $2(N-1)$ equations for both poles and there are 3(N-1) parameters. If measurements are carried out in equally distributed $\mathrm{N}-1$ meridians and in a one equator, then the number of available equations is $2(N-2)$ for both poles and $N-1$ for crossing points of the meridians and the equator. Therefore, $3 N-3$ equations are obtained, which is exactly the same number as the number of unknown parameters.

The general task of profile matching can be formulated with the use of an indicator representing the sum of squares of differences between radii in crossing points of the trajectory. The indicator is described by the (2):

$$
\begin{aligned}
& J_{\text {match }}=\frac{1}{2} \sum_{i=1}^{M} \sum_{j=i}^{M}\left(r_{\text {real }, i}\left(\varphi_{1, i, j}^{\prime}\right)-r_{\text {real }, j}\left(\varphi_{2, i, j}^{\prime}\right)\right)^{2}= \\
& =\frac{1}{2} \sum_{i=1}^{M} \sum_{j=i}^{M}\left(r_{\text {meas }, i}\left(\varphi_{1, i, j}^{\prime}\right)+\Theta\left(\varphi_{1, i, j}^{\prime}\right) p_{\text {match }, i}-r_{\text {meas }, j}\left(\varphi_{2, i, j}^{\prime}\right)-\Theta\left(\varphi_{2, i, j}^{\prime}\right) p_{\text {match }, j}\right)^{2} .
\end{aligned}
$$

In the above equation $\varphi_{1, i, j}^{\prime}$ and $\varphi_{2, j, i}^{\prime}$ are coordinates $\varphi^{\prime}$ of the $i$ and $j$ cross-section in the point of the crossing of sections $i$ and $j$. Obviously, $\varphi_{1, i, j}^{\prime}$ is different from $\varphi_{2, j, i}^{\prime}$, and $\varphi_{1, i, j}^{\prime}$ are coordinates of the point, which is opposite to the point $\varphi_{2, i, j}^{\prime}$. Values $c_{1}, a_{1}, b_{1}$ can be set to zero, i.e. all the profiles will be then matched to the first profile.

It is possible that more than two profiles go through one point. It was assumed that $\varphi_{1, i, i}^{\prime}=\varphi_{2, i, i}^{\prime}=0$. In this sum these profile pairs may be also neglected that are located too close to each other (the length of the vector $v_{o}$ is too small). For convenience of calculations of relevant parameters, the indicator (2) can be written in a matrix form:

$$
J_{\text {match }}=\frac{1}{2} p^{T}{ }_{\text {match }} F p_{\text {match }}+p^{T}{ }_{\text {match }} g+c,
$$


where $p_{\text {match }}=\left[\begin{array}{lllllll}a_{1} & b_{1} & c_{1} & \ldots & a_{N} & b_{N} & c_{N}\end{array}\right]$. Parameters of optimal profile matching are obtained from the following equation:

$$
F p_{\text {match }}+g=0 \text {. }
$$

\subsection{Profile matching with calculation of the reference sphere parameters}

The model presented in the previous section can be easily generalized to match profiles and at the same time to calculate the parameters of the reference sphere (and with the use of the same procedure). In the analyzed case the reference sphere was calculated by the least squares method. Considering the equations given in Section 3.2. one can write that parameters of the reference sphere fulfill the following condition:

$$
G p_{\text {ref }}=\sum_{i=1}^{M} \int_{0}^{2 \pi} \Psi\left(\theta_{i}, \varphi_{i}\right) r_{\text {real }, i}\left(\varphi_{i}^{\prime}\right) \eta_{i}\left(\varphi^{\prime}\right) d \varphi^{\prime},
$$

where $r_{\text {sphere }, i}\left(\varphi_{i}^{\prime}\right)$ is the real profile. It is obvious that the real profile is not known accurately. A measured profile $r_{\text {meas }, i}\left(\varphi_{i}^{\prime}\right)$ is equal to:

$$
r_{\text {meas }, i}\left(\varphi^{\prime}\right)=r_{\text {sphere }, i}\left(\varphi^{\prime}\right)-\Theta\left(\varphi^{\prime}\right) p_{\text {match }, i} .
$$

Thus:

$$
\begin{aligned}
& G p_{\text {ref }}=\sum_{i=1}^{M} \int_{0}^{2 \pi} \Psi\left(\theta_{i}, \varphi_{i}\right) r_{\text {meas }, i}\left(\varphi_{i}^{\prime}\right) \eta_{i}\left(\varphi^{\prime}\right) d \varphi^{\prime}+\sum_{i=1}^{M}\left(\int_{0}^{2 \pi} \Psi\left(\theta_{i}, \varphi_{i}\right) \Theta\left(\varphi^{\prime}\right) \eta_{i}\left(\varphi^{\prime}\right) d \varphi^{\prime}\right) p_{\text {match }, i}= \\
& =\rho+\sum_{i=1}^{M} W_{i} p_{\text {match }, i}=\rho+\left[\begin{array}{llll}
W_{1} & W_{1} & \ldots & W_{M}
\end{array}\right] p_{\text {match }}=\rho+W p_{\text {match }},
\end{aligned}
$$

where $W$ is the matrix whose dimensions are $3 M x 4$. The expression $W_{i}$ can be easily calculated for any cross-section of the spherical element.

Because such matching parameters are sought for whom the calculated profiles will be a deviation from the reference sphere, one can assume that $p_{r e f}=0$, which gives:

$$
g+W p_{\text {match }}=0 .
$$

As a result, optimum parameters can be obtained from optimization conditions for a Lagrange function:

$$
L=\frac{1}{2} p_{\text {match }}^{T} F p_{\text {match }}+p^{T}{ }_{\text {match }} g+\lambda^{T}\left(\rho+W p_{\text {match }}\right),
$$

thus:

$$
\left(\begin{array}{cc}
F & W^{T} \\
W & 0
\end{array}\right)\left(\begin{array}{c}
p_{\text {match }} \\
\lambda
\end{array}\right)+\left(\begin{array}{l}
g \\
\rho
\end{array}\right)=0 .
$$

It is obvious that it is not necessary to calculate matrix $G$ directly.

After calculation of the matrix $W$ it is very easy to calculate values of matching parameters.

\section{Verification of the concept of profile matching}

The developed concept of profile matching has been tested for a spherical surface shown in Fig. 4. 


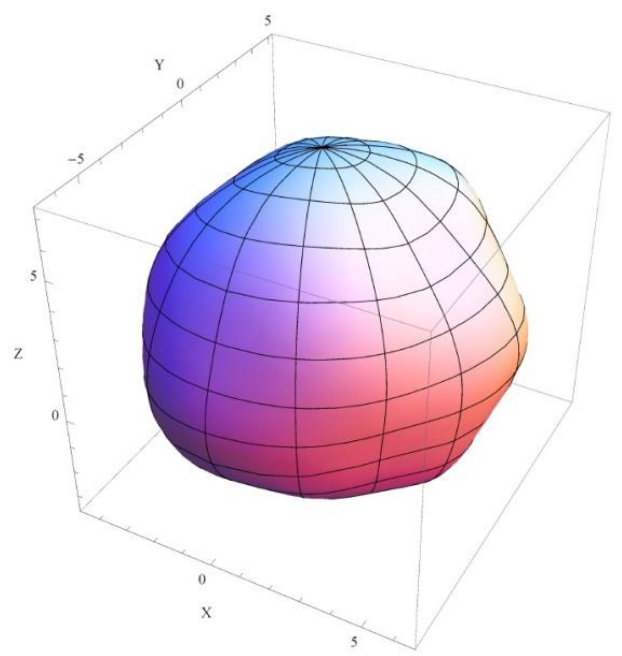

Fig. 4. Investigated spherical surface.

At the first stage of the simulations, the orthogonal strategy was applied (i.e. three mutually perpendicular cross-sections are measured). Firstly, data of investigated surface had been entered, parameters of measurement strategy had been determined, deviations of profiles were calculated and finally the coordinates of the crossings of profiles were established.

Next, measured profiles have been defined. It was assumed that each profile includes some random values of parameters $(a, b, c)$ in relation to the profile calculated with respect to the reference sphere. It was conducted by the following Mathematica instructions:

$a=\{1,0,0\} ; b=\{0,1,0\} ; c=\{0,0,1\}$;

$r m=T a b l e[\Delta r r[[i]]-a[[i]] \operatorname{Cos}[\varphi t]-b[[i]] \operatorname{Sin}[\varphi t]-c[[i]],\{i, M\}] ;$

ProfilePlot [r_, m,rad_]:=Module $\left[\left\{\mathrm{M}_{-} \mathrm{ms}\right\}\right.$;

$\mathrm{M}=$ Length $[\mathrm{m}] ; \mathrm{ms}=\operatorname{Map}[\mathrm{sub}, \mathrm{m}]$;

Show [Table [ParametricPlot3D [Spheric2Cart [\{r[[i]]+rad, $\theta$ s/.ms [ [i] ], $\varphi s / . m s[[$

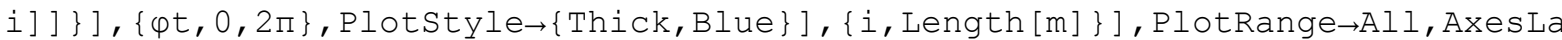
bel $\rightarrow\{$ "X", "Y","Z"\}] ]

A diagram showing measured roundness profiles is shown in Fig. 5.

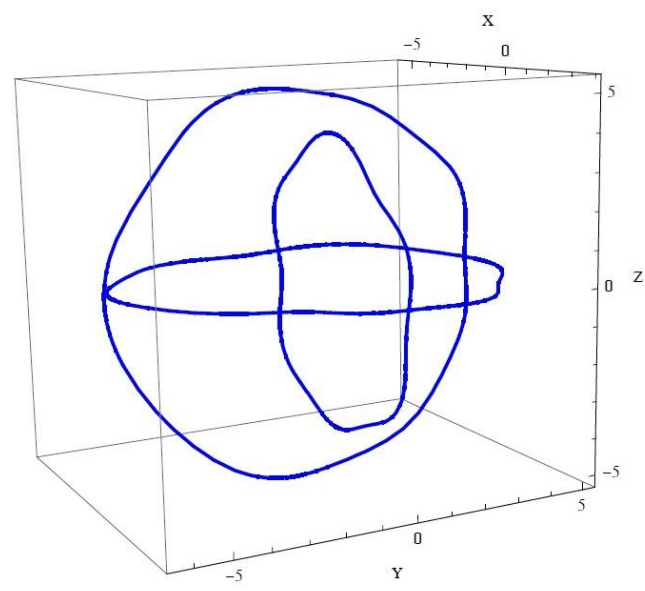

Fig. 5. Measured roundness profiles.

To test if crossing points of the profiles have been calculated correctly, the following instructions were used:

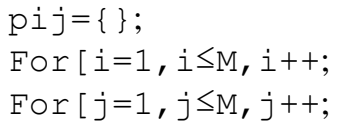


If $[i==j$, Continue []$]$;

$\{\varphi i j, \varphi j i$, norm $\}=$ GetCrosSectionPhase $[m[[i]], m[[j]]]$;

pij=pij Join \{Sphere [ $\{\operatorname{Cos} \varphi \sin \theta \operatorname{s}, \sin \varphi \sin \theta s, \operatorname{Cos} \theta s\} / . m s[[i]]$

/. $\varphi t \rightarrow \varphi i j, 0.05]\} ;]]$

Show [MethodVisualization [m], Graphics3D [ \{Green,pij\}] ].

With the use of the instructions given above a diagram shown in Fig. 6 was obtained.

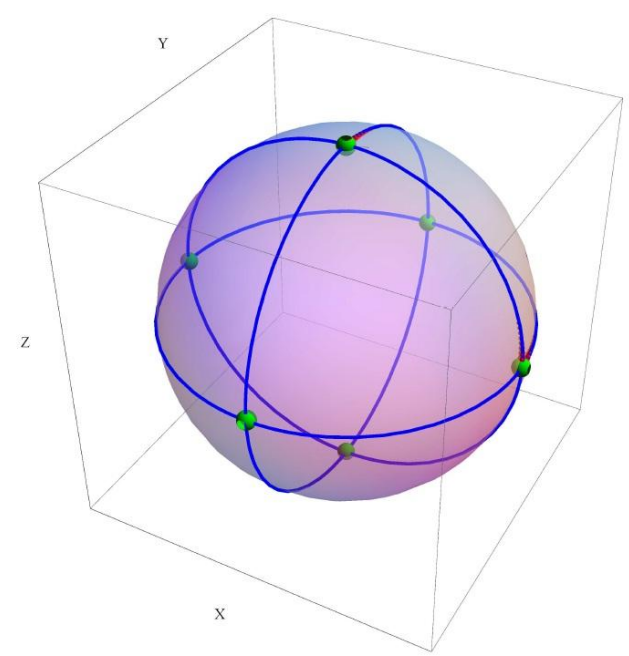

Fig. 6. Crossing points of profiles determined with the use of the function GetCros SectionPhase.

The next operation was calculation of the matching parameters that was conducted by the following instructions:

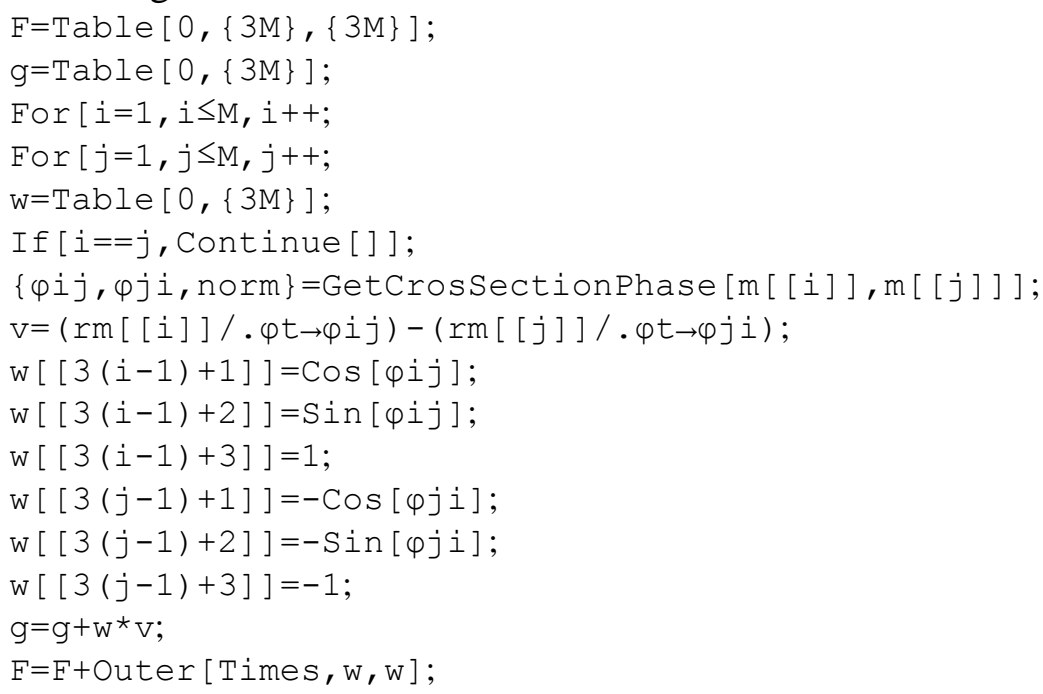

Results obtained by applying the instructions given above show that the number of parameters is too high if the row of the matrix $F$ is lower than its dimension. It is quite simple to understand, if one considers the following case: let us add an equation of a certain sphere $R^{\prime}(\theta, \varphi)=r_{0}^{\prime}+e^{\prime}{ }_{x} \cos \varphi \sin \theta+e^{\prime}{ }_{y} \sin \varphi \sin \theta+e^{\prime}{ }_{z} \cos \theta$ to all measured profiles. It is obvious that this addition will not change conditions of profile matching. So, one can conclude that there are four parameters in excess among assumed matching parameters. It is quite easy to check that the difference between the row of the matrix $\mathrm{F}$ and its dimension is equal to four, indeed. It is obvious that one can simply assume that parameters $a_{1}, b_{1}, c_{1}$ are equal to zero (i.e. assume that all remaining profiles should be matched to the first one). However, finding another parameter to remove can sometimes be difficult, because it can depend on the applied measuring strategy. In the analyzed case one can assume that $a_{2}=0$. Supposedly, it can be 
conducted in different ways, but it will depend on the measuring strategy. For example, in the case of an orthogonal strategy, parameter $b_{2}$ cannot be rejected.

One of the solutions can be found by applying the function Linearsolve of the Mathematica software:

pmatch=-LinearSolve $[\mathrm{F}, \mathrm{g}] / /$ Chop

In the given example following parameters of profile matching were obtained: $a_{1}=0.5, b_{1}=-0.5, c_{1}=-0.333, a_{2}=0, b_{2}=0.5, c_{2}=-0.333, a_{3}=-0.5, b_{3}=0, c_{3}=0.667$.

Figure 7 shows profiles after their matching with the use of obtained parameters.

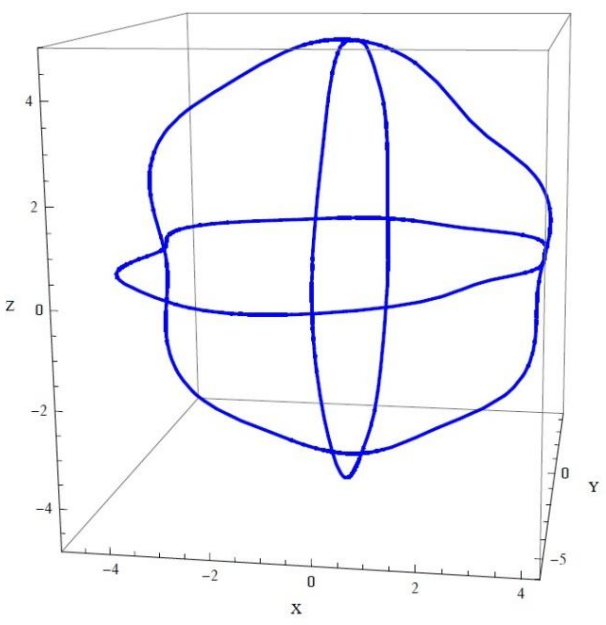

Fig. 7. Profile matching with the use of parameters obtained by applying the function LinearSolve.

Additional research showed that if four parameters of the vector $p_{\text {match }}$ are equal to zero, then a slightly different solution is obtained: $a_{1}=0, b_{1}=0, c_{1}=0, a_{2}=0, b_{2}=1, c_{2}=0, a_{3}=-1, b_{3}=0$, $c_{3}=1$. This is why in this case the method of profile matching presented in Section 3.3 should be applied. By applying equations given in Section 3.3 one can avoid assuming that some parameters should be equal to zero but sets additional conditions that should be fulfilled.

In this way it is possible to obtain the deviation of the profile from the reference sphere and the profile matching in one operation. By applying the equations given in Section 3.3 the following values of matching parameters were obtained: $a_{1}=1, b_{1}=0, c_{1}=0, a_{2}=0, b_{2}=1, c_{2}=0$, $a_{3}=0, b_{3}=1, c_{3}=1$. Matched profiles on the background of the calculated reference sphere are shown in Fig. 8.

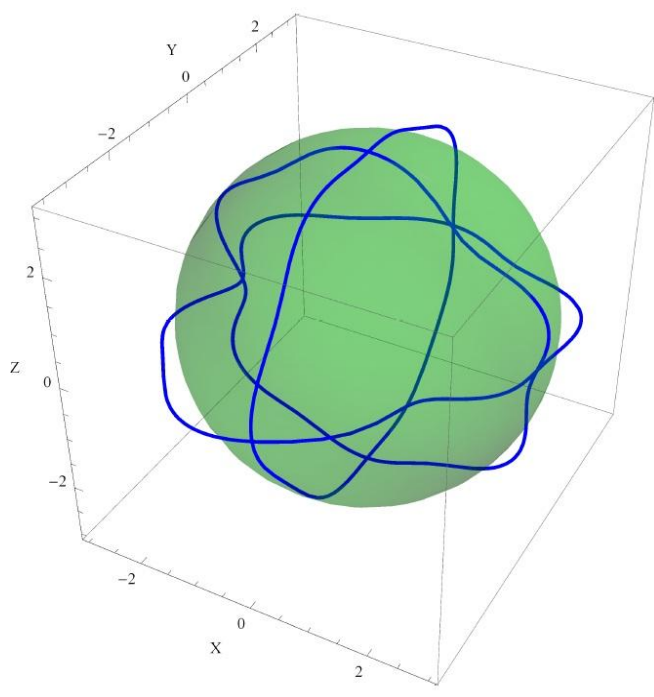

Fig. 8. Matched profiles on the background of the calculated reference sphere. 
The model developed in Section 3.3 was also verified in the case of applying the "Cage" strategy. Fig. 9 shows measured roundness profiles of the surface presented in Fig. 4. In order to test developed equations accurately, there were random values of the mean radius and an eccentricity added to each profile.

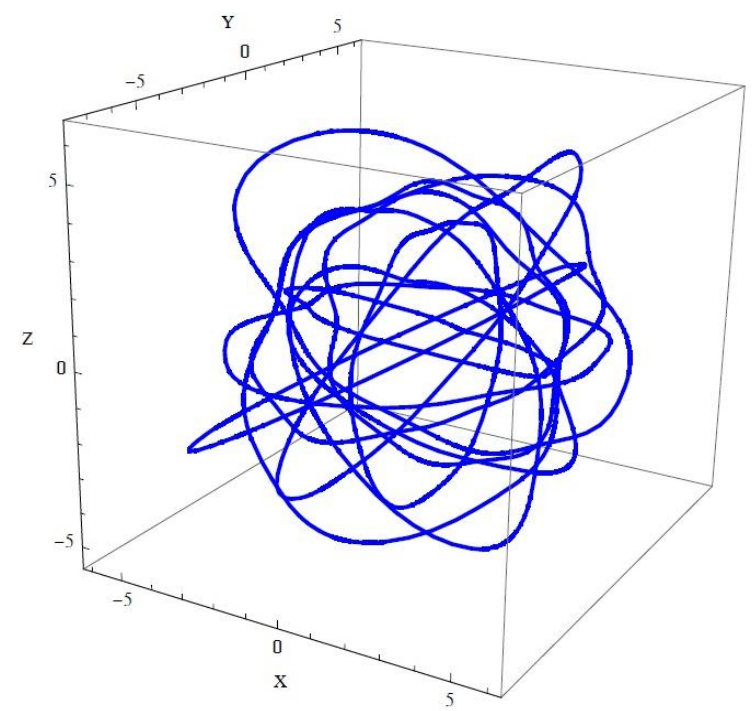

Fig. 9. Roundness profiles obtained by the "Cage" strategy with added random values of mean radii and eccentricity.

After applying equations given in Section 3.3, values of matching parameters were obtained that allow to match the profiles correctly. It is confirmed by diagrams shown in Fig. 10 and in Fig. 11.

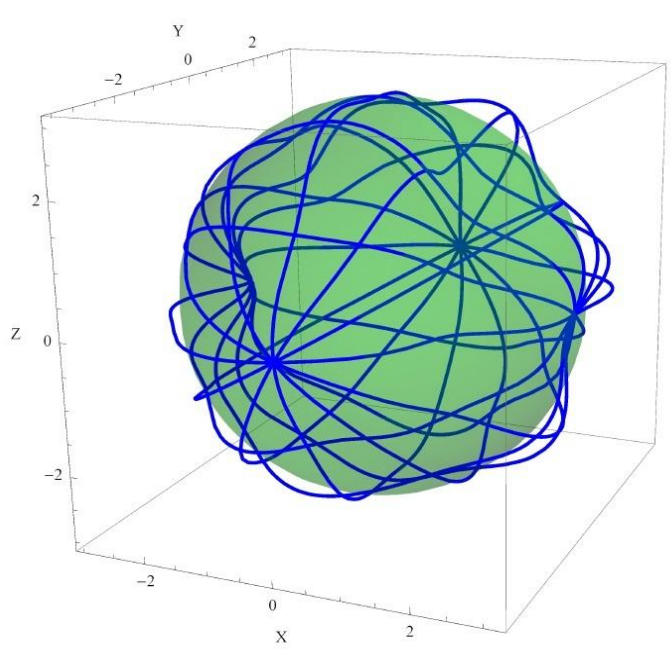

Fig. 10. Profiles obtained by the "Cage" strategy after matching and calculation of the reference sphere.

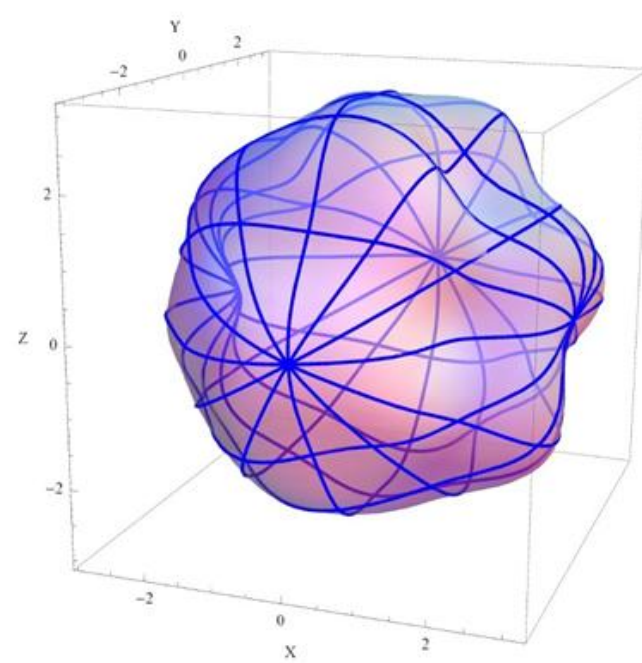

Fig. 11. Profiles obtained by the "Cage" strategy after the matching in the background of an approximated surface.

\section{Summary}

Form errors of spherical machine parts are usually evaluated on the basis of measurement results of roundness profiles in a few selected cross-sections of the investigated element. Such approach allows a rough evaluation of form errors only, particularly if there are significant 
local irregularities on the surface. This is the reason why at the Kielce University of Technology a new concept of sphericity measurements has been developed.

The concept permits to measure roundness profiles along such trajectories that assure accurate coverage of the investigated surface with a network of sampling points. It is based upon application of a typical radius-change measuring instrument equipped with a specialpurpose unit for accurate positioning of spherical elements. The developed concept required the solution of a series of problems related to the applied measurement method. One of these problems was matching of measured roundness profiles. This problem was successfully solved by development of mathematical equations that allow calculation of matching parameters. In the research work the least square method was applied. The developed equations were tested through computer simulations with the use of procedures written in the language of Mathematica software. Results of the simulation confirmed that the developed concept of profile matching is correct.

Thanks to solving the problem of profile matching it was possible to carry out further research activities, including calculation of reference sphere parameters and sphericity deviations and then to test the developed concept experimentally with the use of a test stand. The results of further research work the authors are going to present in future papers.

\section{Acknowledgements}

This work was supported by the Ministry of Science and Higher Education, Poland (grant no. N R0300 2510).

\section{References}

[1] Zawada-Tomkiewicz, A., Ściegienka, J. (2011). Monitoring of a micro-smoothing process with the use of machined surface images. Metrol. Meas. Syst., 18(3), 419-428.

[2] Thalmann, R., Spiller, J. (2005). A primary roundness measuring machine. Recent Developments in Traceable Dimensional Measurements III, Proc. SPIE, 5879.

[3] Kanada, T. (1997). Estimation of sphericity by means of statistical processing for roundness of spherical parts. Precision Engineering, 20(2), 117-122.

[4] Kanada, T. (1995). Evaluation of spherical form errors - Computation of sphericity by means of minimum zone method and some examinations with using simulated data. Precision Engineering, 17(4), 281-289.

[5] Gleason, E., Schwenke, H. (1998). A spindless instrument for the roundness measurement of precision spheres. Precision Engineering, 22(1), 37-42.

[6] Udupa, G., et al. (1998). Assessment of surface geometry using confocal scanning optical microscope. Mechatronics, 8, 187-215.

[7] Bartl, G., et al. (2010). Interferometric determination of the topographies of absolute sphere radii using the sphere interferometer of PTB. Measurement Science and Technology, 21(11), 115101.

[8] Halkaci, H. S., Mavi, Ö., Yigit, O. (2007). Evaluation of form error at semi-spherical tools by use of image processing. Measurement, 40(9-10), 860-867.

[9] Chen, L.C. (2007). Automatic 3D surface reconstruction and sphericity measurement of micro spherical balls of miniaturized coordinate measuring probes. Measurement Science and Technology, 18, 1748-1755.

[10] Samuel, G.L., Shunmugam, M.S. (2003). Evaluation of circularity and sphericity from coordinate measurement data. Journal of Materials Processing Technology, 139(1-3), 90-95.

[11] Nafi, A., Mayer, J.R.R., Woźniak, A. (2011). Novel CMM-based implementation of the multi-step method for the separation of machine and probe errors. Precision Engineering, 35(2), 318-328.

[12] Janecki, D., Stępień, K., Adamczak, S. (2010). Investigating methods of mathematical modelling of measurement and analysis of spherical surfaces In Proc. of the $X$ International Symposium on Measurement and Quality Control, 6-9 Sept., 2010, Osaka, Japan. 
D. Janecki, S. Adamczak, K. Stępień: PROBLEM OF PROFILE MATCHING IN SPHERICITY MEASUREMENTS ...

[13] Kunis, S., Potts, D. (2003). Fast Spherical Fourier algorithms. Journal of Computational and Applied Mathematics, 161(1), 75-98. 